\title{
Reconstrucción robotizada del patrimonio arquitectónico chileno en madera
} Robotic reconstruction of Chilean wooden architectural heritage

\author{
Luis Felipe González Böhme \\ Universidad Técnica Federico Santa María, Chile \\ luisfelipe.gonzalez@usm.cl \\ Francisco Javier Quitral Zapata \\ Universidad Técnica Federico Santa María, Chile \\ francisco.quitral@usm.cl
}

\author{
Sandro Maino Ansaldo \\ Universidad Técnica Federico Santa María, Chile \\ sandro.maino@usm.cl \\ Marcela Hurtado Saldías \\ Universidad Técnica Federico Santa María, Chile \\ marcela.hurtado@usm.cl
}

\begin{abstract}
We present a proof of concept of parametric 3D models of fully associative geometry and milling tool paths for the robotic machining of traditional timber joints, using a visual robot-programming environment integrated into a popular CAD software. A representative sample of traditional timber joints was obtained from a field survey conducted in Valparaíso, Chile. Each specimen was theoretically validated in nearly half a hundred carpentry treatises and manuals corresponding to the historical period in which the surveyed buildings were built. Parametric robotic milling prototypes were experimentally validated in manufacturing process using two industrial robots with different spindles and cutting tools.
\end{abstract}

Keywords: Mecanizado robótico; Programación visual de robots; Patrimonio arquitectónico; Carpintería de armar; Uniones carpinteras

\section{Introducción}

La ciudad puerto de Valparaíso en Chile oculta a simple vista un valioso patrimonio arquitectónico en la estructura portante de pisos, muros y cubiertas de muchos de sus edificios. Se trata de entramados de madera con uniones carpinteras de geometría compleja, laboriosamente labradas y ensambladas entre fines del siglo XIX y principios del XX por carpinteros de armar chilenos y extranjeros con conocimiento ancestral y herramientas de vanguardia para la época. Las uniones carpinteras se distinguen de las uniones mecánicas por transmitir los esfuerzos de un madero a otro a través de formas como cajas o rebajes y espigas o llaves, talladas y ensambladas recíprocamente, reduciendo el aporte de hierro o acero al mínimo y con el único propósito de impedir que la unión se desarme (Arriaga, Iñiguez, Esteban, Argüelles, \& Fernández, 2011). La función de una unión carpintera puede ser la de empalmar, ensamblar o acoplar, es decir, unir miembros de un entramado de madera por sus testas para alcanzar mayor longitud, unir miembros en un ángulo determinado o unir miembros por sus caras para obtener mayor sección. La variedad de uniones carpinteras tradicionales es amplia, destacándose las japonesas por sobre las europeas debido a su nivel de complejidad geométrica superior. Ver Sumiyoshi and Matsui (1991) y Gerner (1992) para una visión general de uniones carpinteras tradicionales de la cultura japonesa y germánica respectivamente. Lamentablemente, el patrimonio arquitectónico en madera de Valparaíso sucumbe de manera sostenida a los incendios, termitas, hongos, condiciones meteorológicas y ambientales. La insuficiente inversión en su mantenimiento impide conservar enteramente la estructura y envolvente original de muchos edificios con cualidades constructivas o estéticas valorables. Además, la escasez a nivel nacional de carpinteros de armar cualificados impide restaurar de manera regular y adecuada la concepción estructural original de estos edificios y, con ello, conservar o incluso mejorar su capacidad de resistencia -a incendios, sismos y otros factores de deterioro- para la que dichas estructuras fueron previstas originalmente. El remplazo de

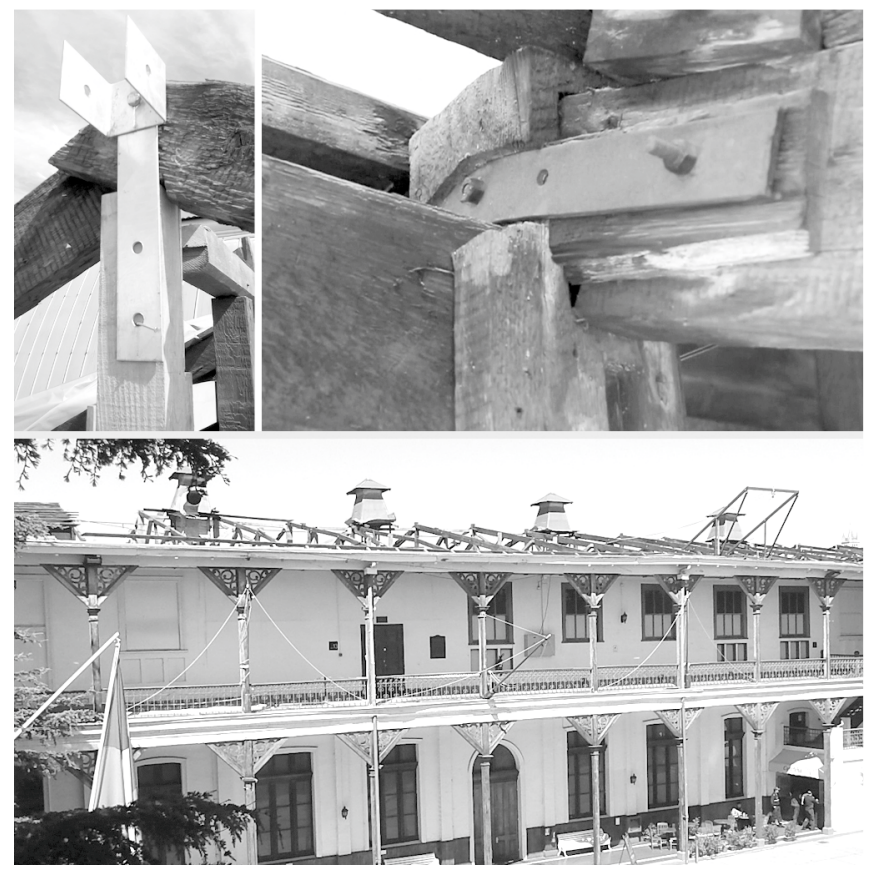

Figura 1: remplazo de uniones carpinteras y herrajes originales (der.) por conectores metálicos ad hoc (izq.) en la restauración de la armadura de cubierta del pabellón central del Museo Marítimo Nacional de Chile, Valparaíso, 2016. 
entramados y uniones carpinteras por productos industriales de materiales y características estructurales muy diferentes de las originales, en general, aumenta inapropiadamente la rigidez y el peso de las estructuras, elevando además los costos de restauración (Parisi \& Piazza, 2002). En Valparaíso es común el remplazo de uniones carpinteras originales por uniones a tope (i.e. sin labrar) fijadas por clavos y tornillos o por conectores metálicos que a veces son fabricados sólo para ese fin, encareciendo aún más los costos de restauración (Figura 1).

La ausencia actual de una normativa exclusiva para las uniones carpinteras en Chile, también desincentiva su aplicación por parte de proyectistas y constructores. La carencia de especificaciones técnicas estandarizadas (i.e. criterios de desempeño) para las uniones carpinteras, la alta demanda de tiempo para su elaboración y el alto costo que generan los errores de manufactura asociados a la total ausencia de formación técnica de nuevos carpinteros de armar cualificados, contribuyen también a descartar su uso en la construcción en general y la restauración en particular. Es, por lo tanto, necesario innovar en la fabricación bajo pedido de uniones carpinteras y piezas de madera de diseño específico (e.g. pasamanos y peldaños de escaleras), a costos competitivos. El mecanizado de formas complejas en madera con robots industriales de 6 o más ejes surge como una alternativa de manufactura flexible, rápida y con baja tasa de errores de manufactura. La pregunta de investigación es ¿cómo implementar la reconstrucción robotizada del patrimonio arquitectónico chileno en madera?
Para responder esta pregunta condujimos de modo pionero en Chile un proyecto de investigación y desarrollo de algoritmos para el mecanizado robótico de uniones carpinteras tradicionales halladas en Valparaíso. Este proyecto de ciencia aplicada comprometió dos resultados de producción: un catálogo de uniones carpinteras empleadas originalmente en la construcción de Valparaíso de fines del siglo XIX a principios del XX y una colección de modelos 3D paramétricos de trayectorias de herramienta adaptativas para el mecanizado de esas uniones carpinteras con un robot industrial (Figura 2).

\section{Robots en la industria creativa}

Un robot industrial es un manipulador multipropósito, reprogramable, controlado automáticamente, programable en 3 o más ejes, que puede estar fijo en un lugar o móvil para su uso en aplicaciones de automatización industrial (ISO, 2012). Actualmente se estiman aproximadamente 230 robots industriales instalados en Chile, número ampliamente superado en Latinoamérica por Brasil con 20.000, México con 17.500 y Argentina con 2.000 unidades (Lohrmann, 2016). En Chile la mayoría de ellos sirve en la minería, algunos pocos en la construcción naval, las industrias de alimentos y bebidas pulpa y papel, vidrio, energía, metal y recientemente también en medicina. Excepto por una empresa que dice utilizar un robot industrial para la limpieza de encofrados, no hay evidencia contundente de aplicación significativa de robots en la industria de la construcción chilena ni menos en la carpintería de armar. Paradójicamente, entre las principales metas del estado de Chile para el año 2025 (CORFO, 2017)

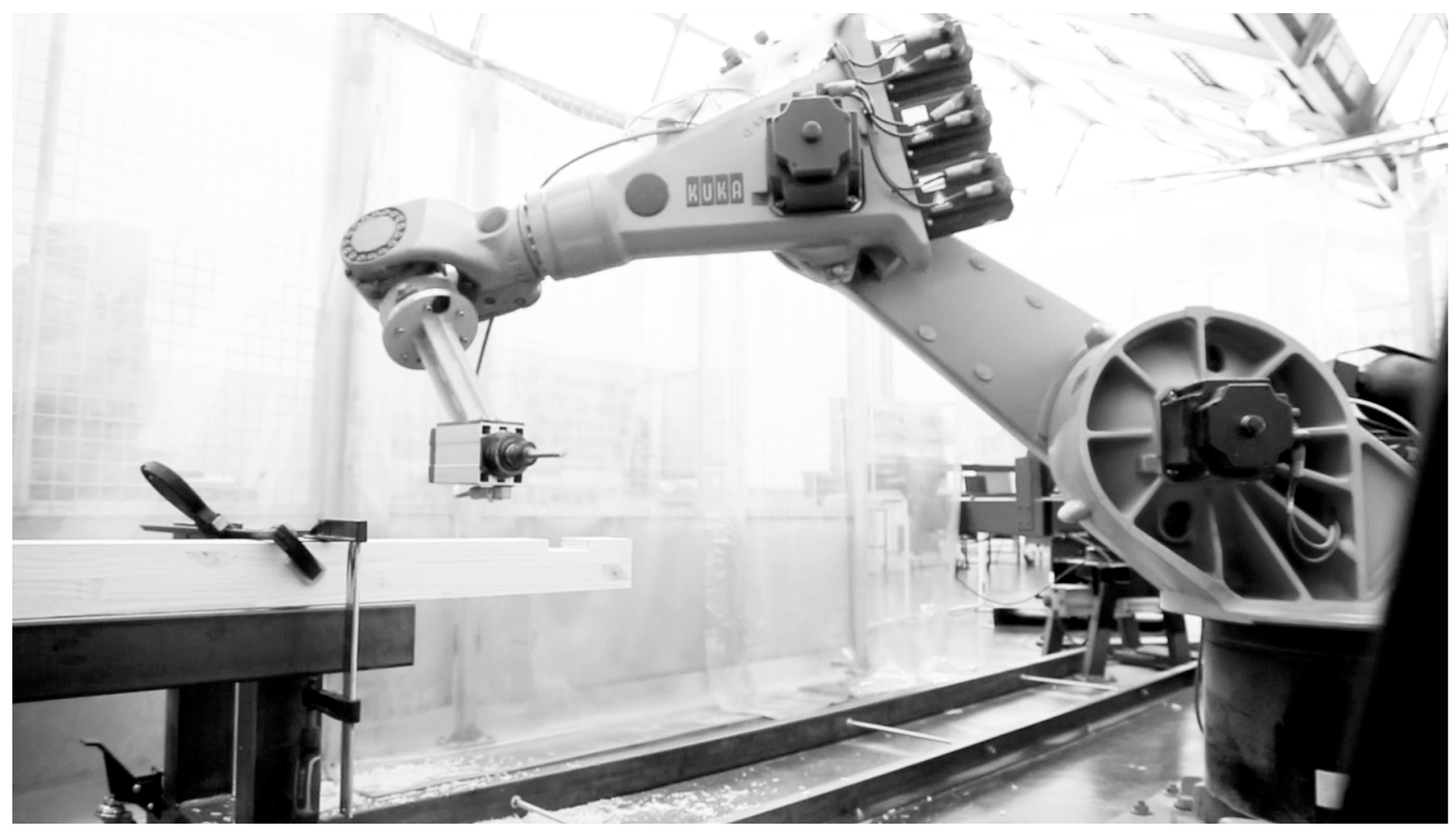

Figura 2: mecanizado de una unión carpintera en una solera superior con un robot KUKA KR 125/2, equipado con un electrohusillo HSD de 4.5KW de potencia, CIMA, UTFSM, 2016 
están aumentar en un 30\% el número de viviendas que se construye en madera, aumentar también en un $30 \%$ las exportaciones de PYMEs de productos más sofisticados de remanufactura en maderas y catalizar el desarrollo de la manufactura avanzada para así frenar la alta desindustrialización que el país viene experimentando ya por décadas.

Aunque los arquitectos suizos Fabio Gramazio y Matthias Kohler (2014) introdujeron el uso de robots industriales en arquitectura en el año 2005, no fue sino hasta después que los arquitectos austriacos Johannes Braumann y Sigrid BrellÇokcan (2011) lanzaran en 2011 el entorno gráfico KUKA|prc de programación offline para robots de la marca alemana KUKA, que cualquier persona sin conocimiento previo de programación de robots industriales pudo acceder a la robótica industrial desde el diseño paramétrico, casi sin necesidad de intermediarios. El diseño paramétrico o variacional, es una técnica que permite instanciar diseños genéricos para una variedad de valores de parámetros y restricciones. La variación o readecuación del modelo 3D paramétrico ocurre de manera automática debido a una red de dependencia entre los parámetros descriptivos de su geometría, especificada por el diseñador. Existen entornos de programación visual cada vez más amigables, que facilitan la especificación de parámetros y restricciones de diseño mediante la manipulación de íconos dentro de una interface gráfica de usuario. Consistentemente, la programación visual, también llamada programación gráfica o programación por íconos utiliza un lenguaje de programación que se expresa en forma de diagramas de nodos, también llamados algoritmos visuales y que es interpretado exclusivamente por la aplicación que aloja al entorno. En programación visual, el algoritmo y el modelo que lo representa son una misma cosa. KUKA|prc y poco después HAL, del arquitecto francés Schwartz (2013) -que ofrece compatibilidad adicional con otras marcas de robots-, han fomentado el potencial de máquina-herramienta de los robots industriales al haber integrado el diseño paramétrico. Ambas aplicaciones se alojan dentro del entorno de programación visual multipropósito Grasshopper, desarrollado por el arquitecto holandés David Rutten. Grasshopper se ofrece como descarga gratuita para ser ejecutada (i.e. interpretada) en una copia licenciada de Rhinoceros (Robert McNeel and Associates), una de las aplicaciones CAD / CAM más populares y avanzadas actualmente. Grasshopper tiene la virtud de beneficiarse de un ecosistema de plug-ins que permite la interacción en tiempo real con software (e.g. Excel, Photoshop, Revit, Ecotect, etc.) o hardware externo (e.g., KUKA Robot Controller $\mathrm{KRC}$, Arduino, Kinect, etc). Además, una creciente comunidad a nivel mundial de usuarios muy activos de Grasshopper, desarrollan y comparten de manera gratuita sus propios algoritmos visuales. Fue precisamente el desarrollo experimental de KUKA|prc lo que nos animó a introducir en 2013 por primera vez en Chile la programación de robots industriales en un taller de arquitectura de la Universidad Técnica Federico Santa María en Valparaíso (Chiarella, González, \& Calvo, 2013).

Los arquitectos y diseñadores Dank and Freißling (2013); Johns and Foley (2014); Robeller, Nabaei, and Yves (2014);
Robeller and Weinand (2016); Schwinn, Krieg, and Menges (2013); Søndergaard et al. (2016); Williams and Cherrey (2016) han hecho en los últimos 4 años una contribución significativa al conocimiento en el campo de la manufactura robótica de estructuras de madera, aunque ninguno ha enfocado su investigación específicamente en la manufactura de uniones carpinteras tradicionales. Sólo Tamke y Thomsen (2008; 2009) habían explorado con anterioridad la aplicación de diseño paramétrico a la producción unitaria de una serie de uniones carpinteras únicas para una estructura liviana de madera, aunque utilizando el software CAD / CAM de HSB y una ensambladora CNC de 5 ejes Hundegger.

\section{Catálogo de uniones carpinteras}

El primer objetivo de esta investigación consistió en indagar la presencia de uniones carpinteras tradicionales en edificios históricos de la ciudad de Valparaíso, catalogarlas y especificar los parámetros descriptivos de su geometría para facilitar su reproducción. Con este fin se realizó el levantamiento en terreno de las características morfológicas y funcionales de 34 casos de uniones carpinteras tradicionales, halladas en entramados horizontales y verticales y 52 casos hallados en armaduras de cubierta de 8 edificios localizados dentro y fuera del sitio UNESCO y del área histórica de Valparaíso. La metodología incluyó el registro métrico, gráfico, fotográfico y fílmico in situ de cada caso, así como la referencia bibliográfica de sus precedentes europeos. Los datos obtenidos del levantamiento en terreno fueron validados mediante la comparación exhaustiva de los 86 casos entre sí y con sus precedentes, descritos en 43 tratados y manuales constructivos para edificaciones con entramado de madera publicados en Alemania, Reino Unido, Francia, Italia y España entre 1792 y 1927, entre ellos los de Barberot (1911); Biston, Boutereau , and Hanus (1842); Cabanié (1864 [1848]); Emy (1841); Gaztelu (1899); Oslet (1890). Se hallaron copias originales de algunos de los documentos consultados en la Biblioteca Nacional de Chile, la Biblioteca Santiago Severin de Valparaíso, las bibliotecas de la Facultad de Arquitectura y Urbanismo de la Universidad de Chile y la Facultad de Arquitectura, Diseño y Estudios Urbanos de la Pontificia Universidad Católica de Chile en Santiago.

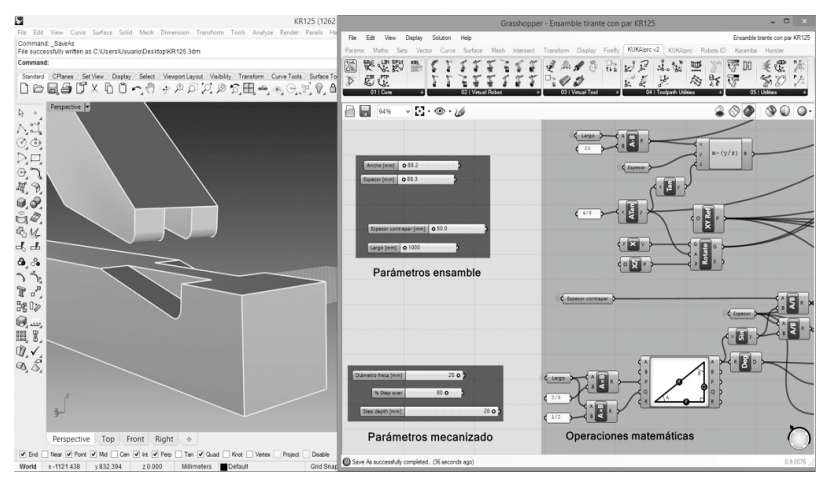

Figura 3: entorno de programación visual de robots industriales KUKA|prc (Braumann \& Brell-Çokcan), alojado en Grasshopper (Rutten), a su vez, integrado en Rhinoceros (Robert McNeel and Associates) 


\section{Modelos de mecanizado robótico}

El segundo, aunque principal, objetivo de esta investigación consistió en producir una colección de modelos 3D paramétricos de uniones carpinteras halladas en Valparaíso, con trayectorias de mecanizado robótico asociativas, es decir, capaces de actualizarse de manera automática cuando el usuario asigne nuevos valores a los parámetros geométricos del modelo 3D de la unión carpintera, de la herramienta o de la celda de trabajo del robot. La idea subyacente es integrar el diseño paramétrico con la programación del mecanizado robótico de uniones carpinteras y producir un paquete de modelos de mecanizado robótico de distintas clases de uniones carpinteras. Con este fin se empleó KUKA|prc (Figura 3) para modelar los parámetros descriptivos, propiedades y restricciones geométricas y topológicas de las uniones carpinteras catalogadas. El logro de este objetivo exigió: el diseño y fabricación de una plataforma móvil para un robot KUKA AGILUS KR6 R900 sixx, dispositivos de montaje de una amoladora METABO de $900 \mathrm{~W}$ de potencia y dos electrohusillos HSD de 1 y $4.5 \mathrm{KW}$ de potencia para el robot AGILUS y para un KUKA KR125/2, así como la modificación de la celda de trabajo de éste último, que incluyó el diseño y fabricación de una mesa de trabajo desplazable y fijable sobre rieles de $6 \mathrm{~m}$ de longitud. También fue necesario ordenar la fabricación de herramental ad hoc.

La restricción auto-impuesta de utilizar únicamente KUKA|prc y Grasshopper para todas las etapas del proceso de diseño a manufactura, nos obligó a desarrollar una estrategia ad hoc para el modelado de piezas y ensamblaje -algo solucionado hace mucho tiempo por las aplicaciones de diseño mecánico. Inspirados por la Cinemática de las Máquinas de Reuleaux (Reuleaux, 1876), acordamos considerar cada unión carpintera como un par constructivo (en lugar de un par cinemático) que está compuesto por dos regiones coincidentes de dos elementos par, cuya forma corresponde en pareja, recíprocamente. Si un tercer o $n$-ésimo elemento par se une a un elemento par de un par constructivo, dentro o muy cerca de esa misma región de conexión, denominamos nudo a esa unión múltiple. Para efectos del modelado, sin embargo, tratamos todas las uniones carpinteras siempre en parejas. Fue necesario para nosotros desarrollar inicialmente esta simple, pero útil, teoría para diseñar las estrategias de modelado $3 d$ de las uniones carpinteras y sus trayectorias de mecanizado con KUKA|prc.

\section{Resultados}

Esta investigación permitió desarrollar una colección de modelos 3D paramétricos de geometría y trayectorias de fresado totalmente asociativas para el mecanizado con robots industriales de 27 clases de uniones carpinteras utilizadas en edificios históricos de la ciudad puerto de Valparaíso, Chile. Para cada clase de empalmes y ensambles descritos en el catálogo producido por esta investigación, se creó un modelo 3D paramétrico editable que permite a un usuario potencial modificar la geometría de la unión carpintera, especificar la herramienta o el modelo de mundo (i.e. la celda de trabajo del robot) e inmediatamente obtener una trayectoria del robot actualizada para mecanizar esa precisa instancia de unión carpintera. El desempeño de cada modelo fue evaluado empíricamente, mediante el mecanizado de instancias de cada una de las 27 clases de uniones carpinteras que catalogamos (Figura 4). Cada modelo se expresa como un diagrama de nodos implementado en KUKA|prc e interpretado por Rhinoceros mediante Grasshopper. KUKA|prc permite simular la celda de trabajo, el robot, la herramienta y su trayectoria dinámicamente con el fin de detectar colisiones 0 singularidades. Además genera los archivos de control numérico en código KRL (KUKA Robot Language) para ser ejecutados directamente en el robot sin post-procesamiento. Así, cuando un modelo paramétrico es instanciado, la salida es doble: la simulación gráfica de la trayectoria del robot y el archivo *.src, propiamente tal, requerido por el robot para realizar la tarea programada por el usuario. Esta colección de modelos 3D paramétricos constituye un producto nuevo, cuyo comportamiento operacional y funcional ha sido validado en condiciones experimentales, utilizando software experimental y comercial, dos robots KUKA de distinta generación (KRC2 y KRC4) y función, así como una amplia variedad de herramental. La serie de experimentos demostró teóricamente una disminución en tiempo y costos del proceso de diseño a manufactura, con una brecha relativa significativa de un $49 \%$ en comparación con su mejor competidor en Chile, es decir, fabricantes de ventanas y puertas con fresadoras de pórtico de 3 ejes. La experimentación en laboratorio incluyó la manufactura robótica de un modelo de entramado de madera para demostración (González \& Quitral, 2016) y una pasarela peatonal (González, Quitral, \& Poblete, 2017).

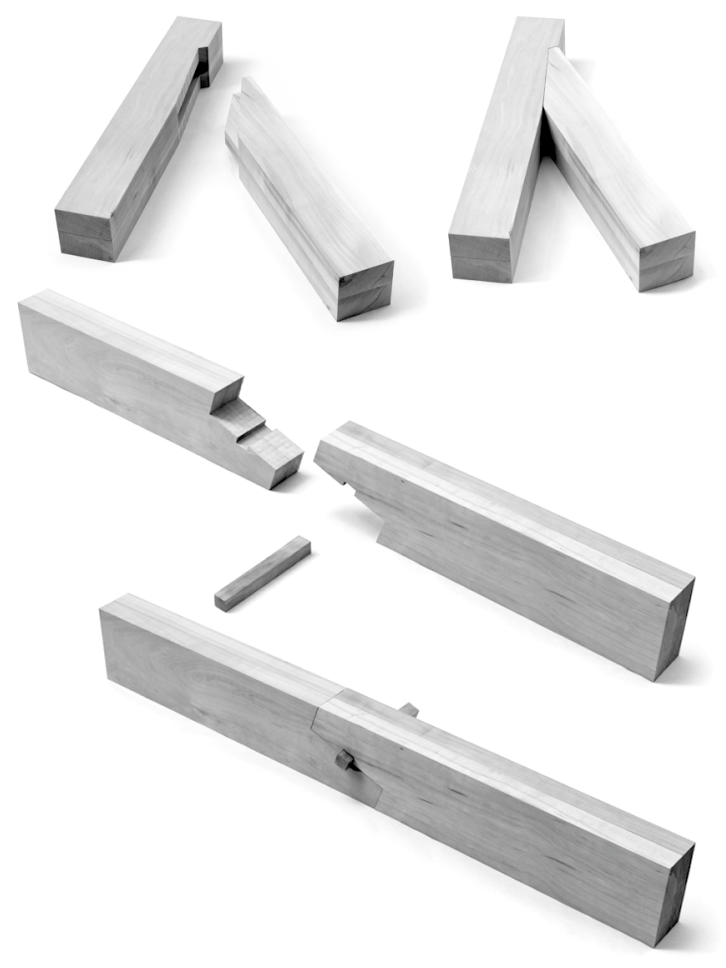

Figura 4: ensamble de espera con cajeado (sup.) y empalme de rayo de júpiter con llave (inf.), hallados en Valparaíso y mecanizados por un robot KUKA KR125/2 con electrohusillo HSD de 4.5KW, fresa helicoidal 20X102X150mm, CIMA, UTFSM, 2016. 


\section{Conclusiones}

El levantamiento en terreno de una muestra representativa compuesta por 86 casos de uniones carpinteras, nos permitió refutar la teoría ampliamente difundida hasta ahora sobre el predominio en Valparaíso del sistema constructivo conocido como balloon frame. Además, la constatación de la presencia de entramados de madera con uniones carpinteras en edificios localizados tanto dentro del sitio UNESCO y el área histórica de Valparaíso como fuera de ambos, nos permiten inferir que hay muchos más casos que requieren ser preservados o reconstruidos. Las uniones carpinteras están atrayendo de nuevo la atención del mundo científico y recuperando parte de su anterior posición en el mercado, principalmente debido a su potencial de sustentabilidad ambiental y económica, que supera en varios aspectos al uso de acero y hormigón en la construcción. Sin embargo, el conocimiento operacional y la destreza manual requeridos para su manufactura, ya se perdieron en muchos lugares del mundo incluido Chile. La robótica ofrece una alternativa competitiva para recuperar y mejorar la técnica del entramado de madera con uniones carpinteras, ya sea para aplicarla en restauración o en obra nueva.

Los modelos desarrollados y validados a nivel experimental en esta investigación ofrecen varias ventajas competitivas como, por ejemplo, automatizar la manufactura de uniones carpinteras; reducir los errores de manufactura; asegurar repetibilidad en la producción; industrializar la producción unitaria y de bajo volumen; manufacturar productos en madera más sofisticados, prescindir de la necesidad de crear parámetros o restricciones y en cambio sólo asignar nuevos valores a los parámetros del modelo que describe la clase de unión carpintera que se desee instanciar; obtener en tiempo real actualizaciones automáticas de la trayectoria de la herramienta al modificar los valores de parámetros 0 especificaciones de la herramienta; prescindir del postprocesamiento de datos usualmente requerido por las aplicaciones CAM convencionales; permitir un flujo de trabajo continuo entre la instanciación del modelo geométrico y la simulación de las trayectorias requeridas para su mecanizado; acelerar y abaratar todo el proceso desde el diseño hasta la manufactura. Además, nuestros experimentos demostraron que KUKA|prc ofrece la posibilidad de programar tareas sin necesidad de depender mucho de un modelo de mundo bien calibrado para obtener rápidamente resultados aceptables. Esta ventaja, combinada con el empleo de un robot ligero como, por ejemplo, el AGILUS que sólo pesa $50 \mathrm{~kg}$, abre un camino hacia la carpintería robótica en obra.

El enfoque original de KUKA|prc en la creación de trayectorias de forma libre para la producción unitaria o en lotes pequeños de piezas únicas, hacen que el modelado procedural de uniones carpinteras -es decir, de pares constructivos y especialmente de nudos- resulte complicado y consuma mucho tiempo. Esta conclusión sugiere orientar nuestro trabajo futuro hacia la integración del modelado de piezas $y$ ensamblaje que corresponde al enfoque de las aplicaciones de diseño mecánico actuales. La integración de esta técnica facilitará la especificación de posición y orientación de cada elemento par de una unión carpintera o de un nudo, así como la verificación de interferencias y la definición de ajuste exacto entre cajas o rebajes y espigas o llaves.

\section{Agradecimientos}

Esta investigación identificada con el código FONDEF ID14I10378, fue financiada por el Fondo para la Promoción del Desarrollo Científico y Tecnológico, administrado por la Comisión Nacional de Investigación Científica y Tecnológica de Chile (CONICYT) entre 2015 y 2017. Para obtener más información, por favor visite: vimeo.com/199477171

Queremos agradecer especialmente a los profesores Eugenio González, Raúl Stegmaier y Daniel Rodríguez y a Maximiano López del Centro Integrado de Manufactura y Automatización (CIMA) de la Universidad Técnica Federico Santa María.

\section{Referencias}

Arriaga, F., Iñiguez, G., Esteban, M., Argüelles, R., \& Fernández, J. L. (2011). Diseño y cálculo de uniones en estructuras de madera. Madrid, España: MADERIA Sociedad Española de la Madera.

Barberot, J. E. C. É. (1911). Traité pratique de charpente. Paris: Ch. Beranger.

Biston , V., Boutereau , C., \& Hanus, P. A. (1842). Nouveau manuel complet du charpentier. Paris: La Librarie Encyclopedique de Roret.

Braumann, J., \& Brell-Çokcan, S. (2011, 13-16 October). Parametric Robot Control: Integrated CAD/CAM for architectural design. Paper presented at the Proceedings of the 31st Annual Conference of the Association for Computer Aided Design in Architecture (ACADIA), Calgary/Banff, Alberta, Canada.

Cabanié, B. (1864 [1848]). Charpente générale théorique et pratique (2 tomos). Paris: Gauthier-Villar.

CORFO. (2017). Transforma: Economía productiva y diversificada Retrieved 13 de Junio, 2017, from www.chiletransforma.cl

Chiarella, M., González, L. F., \& Calvo, C. (2013, 20-22 November). Robots: automatización en diseño y construcción para la enseñanza de arquitectura. Paper presented at the Proceedings of the 17th SIGraDi Conference, Valparaíso, Chile.

Dank, R., \& Freißling, C. (2013). The framed pavilion: Modeling and producing complex systems in architectural education. In S. Brell-Çokcan \& J. Braumann (Eds.), Rob / Arch 2012: Robotic Fabrication in Architecture, Art and Design (pp. 238-247). Vienna, Austria: Springer Wien New York.

Emy, A.-R. (1841). Traité de l'Art de la Charpenterie. Bruxelles: Meline, Cans.

Gaztelu, L. (1899). Carpintería de armar. Madrid: De Bailly-Bailliere e hijos.

Gerner, M. (1992). Handwerkliche Holzverbindungen der Zimmerer. Stuttgart, Germany: Deutsche Verlags-Anstalt DVA.

González, L. F., \& Quitral, F. (Writers) \& C. León \& J. Cubillos (Directors). (2016). Robotically milled timber frame prototype, Creative robotics at UTFSM. Valparaíso, Chile: https://vimeo.com/201582785.

González, L. F., Quitral, F., \& Poblete, D. (Writers) \& Kristofferson \& D. Poblete (Directors). (2017). Leonardo da Vinci's self- 
SIGraDi 2017, XXI Congreso de la Sociedad Ibero-americana de Gráfica Digital

22 - 24 Noviembre, 2017 - Concepción, Chile.

supporting bridge machined by an industrial robot, Creative robotics at UTFSM. Valparaíso, Chile: https://vimeo.com/199476002.

Gramazio, F., \& Kohler, M. (2014). The robotic touch: How robots change architecture. Zürich, Switzerland: Park Books.

ISO. (2012). ISO Standard 8373:2012, Robots and robotic devices Vocabulary 2.9 Industrial robot: International Organization for Standardization.

Johns, R. L., \& Foley, N. (2014). Bandsawn bands feature-based design and fabrication of nested freeform surfaces in wood. In W. McGEE \& M. Ponce de León (Eds.), Robotic Fabrication in Architecture, Art and Design 2014 (pp. 17-32). Switzerland: Springer.

Lohrmann, E. (2016). Cuando la imaginación queda corta: Industria avanzada, la Industria que viene. Charla del Director de canal de ventas para África, Europa, Latinoamérica y Oriente Medio de KUKA Roboter GmbH, sostenida en el XVII Foro Anual de la Industria 2016, organizado por la Asociación de Industriales Metalúrgicos y Metalmecánicos, ASIMET. Santiago, Chile.

Oslet, G. (1890). Traité de charpente en bois. Paris: H. Chairgrasse fils

Parisi, M. A., \& Piazza, M. (2002). Seismic behavior and retrofitting of joints in traditional timber roof structures. Soil Dynamics and Earthquake Engineering, 22(9-12), 1183-1191.

Reuleaux, F. (1876). The kinematics of machinery (A. B. W. Kennedy, Trans.). New York, USA: Macmillan \& Co.

Robeller, C., Nabaei, S. S., \& Yves, W. (2014). Design and fabrication of robot-manufactured joints for a curved-folded thin-shell structure made from CLT. In W. McGee \& M. Ponce de Leon (Eds.), Robotic Fabrication in Architecture, Art and Design 2014 (pp. 67-81). Switzerland: Springer.

Robeller, C., \& Weinand, Y. (2016). Fabrication-aware design of timber folded plate shells with double through tenon joints. In D. Reinhardt, R. Saunders \& J. Burry (Eds.), Robotic Fabrication in
Architecture, Art and Design 2016 (pp. 167-177). Switzerland: Springer.

Schwartz, T. (2013). HAL: Extension of a visual programming language to support teaching and research on robotics applied to construction. In S. Brell-Çokcan \& J. Braumann (Eds.), Rob / Arch 2012: Robotic Fabrication in Architecture, Art and Design (pp. 92-101). Vienna, Austria: Springer Wien New York.

Schwinn, T., Krieg, O. D., \& Menges, A. (2013). Robotically fabricated wood Plate Morphologies: Robotic prefabrication of a biomimetic, geometrically differentiated, lightweight, finger joint timber plate structure. In S. Brell-Çokcan \& J. Braumann (Eds.), Rob ( Arch 2012: Robotic Fabrication in Architecture, Art and Design (pp. 48-61). Vienna, Austria: Springer Wien New York.

Søndergaard, A., Amir, O., Eversmann, P., Piskorec, L., Stan, F., Gramazio, F., \& Kohler, M. (2016). Topology optimization and robotic fabrication of advanced timber space-frame structures. In D. Reinhardt, R. Saunders \& J. Burry (Eds.), Robotic Fabrication in Architecture, Art and Design 2016 (pp. 191-203). Switzerland: Springer.

Sumiyoshi, T., \& Matsui, G. (1991). Wood joints in classical Japanese architecture. Japan: Kajima Institute Publishing Co., Ltd.

Tamke, M., \& Thomsen, M. R. (2008 September 17-19th ). Designing parametric timber. Paper presented at the 26th eCAADe Conference, Antwerpen, Belgium.

Tamke, M., \& Thomsen, M. R. (2009, June 17-19th). Digital wood craft. Paper presented at the 13th International CAAD Futures Conference, Montréal, Canada.

Williams, N., \& Cherrey, J. (2016). Crafting robustness: Rapidly fabricating ruled surface acoustic panels. In D. Reinhardt, R. Saunders \& J. Burry (Eds.), Robotic Fabrication in Architecture, Art and Design 2016 (pp. 295-303). Switzerland: Springer. 